\title{
Genetic affinities of central China populations
}

\author{
H.Y. Zhou ${ }^{1 *}$, H.W. Wang ${ }^{2 *}$, S.N. Tan ${ }^{1}$, Y. Chen ${ }^{1}$, W.L. Wang ${ }^{3}$, H.X. Tao ${ }^{4}$, \\ Z.C. Yin ${ }^{1}$, Y.H. Zou ${ }^{1}$, S.M. Ouyang ${ }^{1}$ and B. Ni ${ }^{1}$
}

${ }^{1}$ Key Laboratory of Genetics and Birth Health of Hunan Province, Family Planning Institute of Hunan Province, Changsha, China

${ }^{2}$ State Key Laboratory of Genetic Resources and Evolution, Kunming Institute of Zoology, Chinese Academy of Sciences, Kunming, Yunnan Province, China

${ }^{3}$ Department of PLA 65553 Medical Team, Haicheng, Liaoning, China

${ }^{4}$ Department of Medical Laboratory, The 210 Hospital of PLA, Dalian, China

*These authors contributed equally to this study.

Corresponding author: B. Ni

E-mail: zhouhaiyan111@yahoo.com.cn

Genet. Mol. Res. 13 (1): 616-625 (2014)

Received January 10, 2013

Accepted June 6, 2013

Published January 28, 2014

DOI http://dx.doi.org/10.4238/2014.January.28.7

ABSTRACT. Hunan locates in the south-central part of China, to the
south of the middle reaches of the Yangtze River and south of Lake
Dongting. According to the historical records, the peopling of Hunan
by modern human ancestors can ascend to 40 thousand years ago. Thus,
to trace the ancient maternal components can offer further insight into
the origin of south-central China. In this study, we investigated the
mitochondrial DNA of 114 individuals from Hunan Province (including
34 Han, 40 Tujia and 40 Miao). Hypervariable regions I and II of the
mtDNA control region were sequenced, and the relative diagnostic
variations in coding region according to the updated worldwide
phylogeny tree were selected and typed by restriction fragment length
polymorphism analysis or direct sequencing. All individuals were
classified into specific (sub)haplogroups. By comparison with the
surrounding populations, southern China-prevalent haplogroups were
detected with relative higher frequency in the Tujia and Miao ethnic 
populations, such as haplogroup B, with more than $20 \%$, lacking in the Han population, which illustrated its southern origin characters. In addition, we also detected northern of East Asia prevalent haplogroups with a relative higher frequency in Tujia populations than in the Miao and Yao ethnic groups, implying a gene flow from Han populations. However, the language-clustering tendency was supported by our principal component analysis and further genetic estimation results. Han and ethnic groups in central China exhibited specific ancestors related to their closer language affinity, although there was extensively genetic admixture between Han and ethnic groups.

Key words: mtDNA; Origin; Ethnic group; Hunan

\section{INTRODUCTION}

Mitochondrial DNA (mtDNA) harbors a series of molecular properties, lack of recombination, mode of inheritance, rapid evolutionary rate, and high population-specific polymorphisms, which make mtDNA a useful genetic marker for studies on the population genetics and molecular anthropology (Bandelt et al., 2001; Forster et al., 2002; Pakendorf and Stoneking, 2005). Especially, the updated phylogenetic tree of China with the entire mtDNA genomes (Kivisild et al., 2002; Zhao et al., 2009; Kong et al., 2003b, 2006, 2011), the quality strategy (Bandelt et al., 2001; Parson and Bandelt, 2007), and the coding region variations typing strategy for verifying the haplogroup status (Yao et al., 2004), mtDNA has offered the fundament for tracing the prehistory origin and migration scenarios at maternal aspect. Thus, the more mtDNA datasets from the crucial geographical region, Hunan, south-central China, will offer more data for tracing the migration and expansion of the anatomically modern human to this area.

Hunan is suited in south-central China, with ethnic enclaves including the Miao, Tujia, Dong, Yao, and Han populations belonging to different language groups. According to historical records, Miao, Tujia, Dong, and Yao peoples are the aboriginal residents of Hunan Province, and Han as well as other minorities settled this region during historic periods (Du and Ye, 1994). Hunan is a Province with many ethnic minority groups. By now, 50 ethnic minority groups are present, with a population of 5.2 million, accounting for $8 \%$ of the total population of Hunan. Except for the recent immigration of Han populations, there is still some debate regarding the origin of the major ethnic groups of Hunan Province, central of China, such as Tujia, Miao, and Yao. The prevalent opinion is that the Tujia was mainly the descendants of the ancient Ba People, settling in western Hunan and Hubei Province, and there were subsequent gene exchanges with other ethnic groups. The Miao ethnic group came from the Jiu-Li tribe in the Yellow River basin of central China, as early as 5000 years ago, and later the San-Miao tribe, who pushed southward to the Yangtze River basin. Thus, further analysis of the aboriginal settlers in this region and the Han populations will be helpful to explore the origin of the minorities and to trace the demographic history of the ethnic populations in Hunan Province.

In this article, we studied the mtDNA sequence polymorphisms of 2 ethnic populations and a Han population by amplifying the hypervariable regions I and II of mtDNA. To 
further illustrate the genetic affinity between the populations investigated in this study, we compared the maternal component of the 3 populations with the surrounding groups retrieved from literature. Except for the genetic admixture results of the populations with the northern and southern China prevalent maternal components, it was significant that in the Hunan populations the consistent affinity between the maternal genetic datasets and their languages was detected. Tujia populations have closer genetic affinity with the Han populations for belonging to the Sino-Tibet language family, and the Yao population shows the greatest genetic distance with Han and Tujia populations, in line with the Hmong-Mien language of the Yao ethnic group.

\section{MATERIAL AND METHODS}

\section{Subjects and data collection}

One hundred and fourteen healthy individuals from 3 populations were collected in Hunan China: Han $(\mathrm{N}=34)$, Miao $(\mathrm{N}=40)$, and Tujia $(\mathrm{N}=40)$. The individuals in the study were selected to avoid known maternal relatives, and genetic investigation ascertained that each sample belonged to the locality for at least 3 generations. Informed consent was obtained from every participant.

Comparative mtDNA data from southeast Asia (Figure 1 and Table 1) and southern China were taken from previously published literature (Wen et al., 2004a,b, 2005).

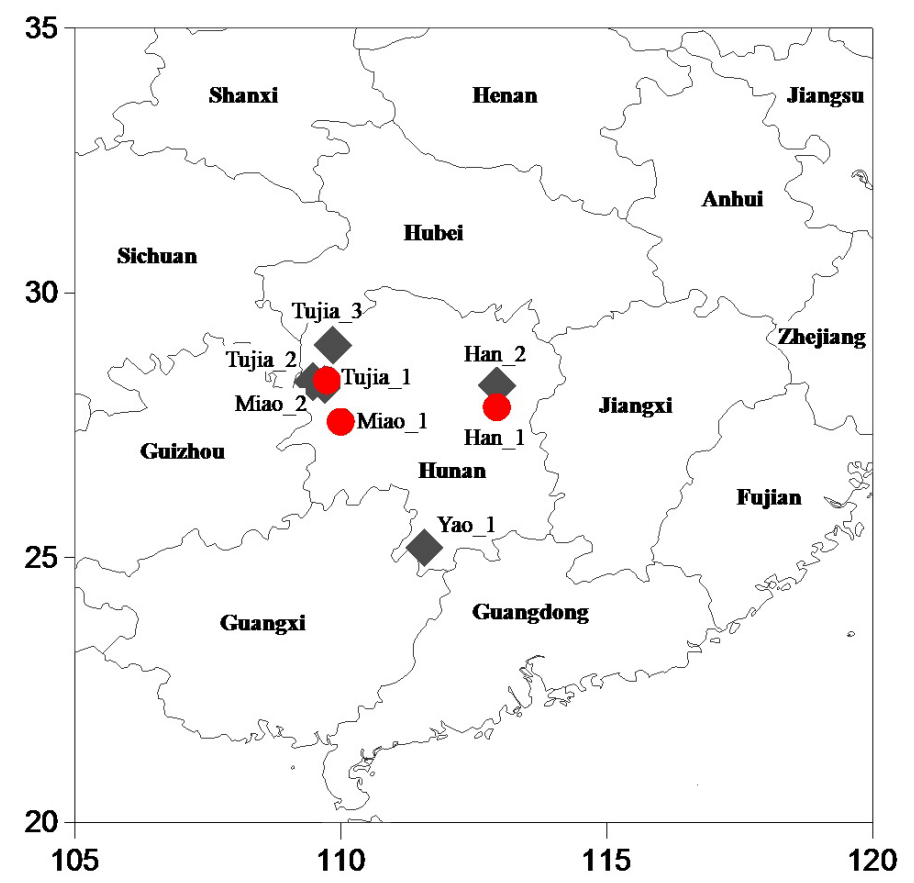

Figure 1. Location of the populations investigated in this study. The populations collected in this study are highlighted with solid circles, and the diamonds represent the populations retrieved from the literature. 


\begin{tabular}{|c|c|c|c|c|c|c|c|}
\hline & Nation & Abbreviation & Location & Country & Language & $\mathrm{N}$ & References \\
\hline 1 & Han & Han_1 & Xiangtan of Hunan & China & Sino-Tibetan & 34 & This study \\
\hline 2 & Miao & Miao 1 & Huaihua of Hunan & China & Hmong-Mien & 40 & This study \\
\hline 3 & Tujia & Tujia_1 & Xiangxi of Hunan & China & Tibeto-Burma & 40 & This study \\
\hline 4 & Han & Han $\overline{2}$ & Changsha of Hunan & China & Sino-Tibetan & 16 & Wen et al., 2004a \\
\hline 5 & Tujia & Tujia 2 & Western Hunan & China & Tibeto-Burma & 66 & Wen et al., 2004b \\
\hline 6 & Tujia & Tujia_3 & Yongshun of Hunan & China & Tibeto-Burma & 31 & Wen et al., 2004b \\
\hline 7 & Miao & Miao_2 & Jishou of Hunan & China & Hmong-Mien & 103 & Wen et al., 2005 \\
\hline 8 & Yao & Yao_- & Jianghua of Hunan & China & Hmong-Mien & 24 & Wen et al., 2005 \\
\hline
\end{tabular}

\section{Genomic DNA extraction, amplification, sequencing, and coding region information typing}

Total DNA was isolated with the standard phenol/chloroform method and stored at $4^{\circ} \mathrm{C}$. Hypervariable regions I and II were amplified with different primer pairs. Detailed information is provided in Table 2. PCR products were purified and sequenced by Shanghai Sangon Biological Engineering \& Services Company. Both strands of the same mtDNA fragment were sequenced to reduce ambiguities in base calling. The strict strategy was followed as suggested (Parson and Bandelt, 2007). The sequences were edited and aligned by the DNASTAR software (DNASTAR) by comparison to the revised Cambridge reference sequence, and the variations were recorded (Andrews et al., 1999). The length of the polymorphisms of the A and C stretches from 16,180 to 16,189 (triggered by the $16,189 \mathrm{~T}$ to $\mathrm{C}$ substitution) was disregarded in the analysis (Yao et al., 2002; Kong et al., 2003a). All of the individuals were screened for the mtDNA 9-bp deletion in the COII/tRNA ${ }^{\text {Lys }}$ intergenic region. According to the HVSI and HVSII variations, all the mtDNA were roughly assigned into the specific haplogroup based on the updated East Asia the worldwide (mtDNA tree Build 15) phylogenetic trees (Kong et al., 2006; van Oven and Kayser, 2009). Subsequently, the specific coding variation(s) were selected and typed by RFLP and/or direct DNA sequencing. The detail information is shown in Table 2.

Table 2. Primers for amplification, sequencing, and RFLP analysis.
\begin{tabular}{llcl}
\hline Primer pairs & Locations in rCRS & Annealing temperature $\left({ }^{\circ} \mathrm{C}\right)$ & Polymorphisms at/in \\
\hline L29/H408 & $8-29 / 429-408$ & 54 & HVSII \\
L15996/H16498 & $15975-15996 / 16517-16498$ & 60 & HVSI \\
L14575/H16048 & $14556-14575 / 16067-16048$ & 53 & $15040,15071,15487 \mathrm{~T}, 15784$ \\
L4210/ H5442 & $4189-4210 / 5461-5442$ & 56 & $4491,5178 \mathrm{~A}$ \\
L4499/H5099 & $4480-4499 / 5118-5099$ & 60 & 4831 HhaI (4833) \\
L9794/H10164 & $9774-9794 / 10181-10164$ & 60 & 9824 HinfI (9824) \\
L3179/H3674 & $3160-3179 / 3693-3674$ & 59 & 3391 HaeIII (3394) \\
L394/H902 & $375-394 / 922-902$ & 60 & 663 HaeIII (663) \\
L8215/H8297 & $8196-8215 / 8316-8297$ & 57 & $9-b p$ deletion \\
L13209/H13458 & $13209-13229 / 13458-13439$ & 59 & 13262 AluI (13262) \\
\hline
\end{tabular}

rCRS $=$ revised Cambridge reference sequence.

\section{Data analysis}

The mtDNAs of 5 Hunan populations (including 1 Han, 2 Tujia, 1 Miao, and 1 Yao group) were retrieved from the literature (Wen et al., 2004a,b, 2005), and the individuals were assigned to specific haplogroup by comparing the East Asian mtDNA data using a similar strategy (Yao et al., 2004). The haplogroup distribution frequencies for each of the 3 populations were calculated (Table 3). Principal component analysis was performed by taking the 


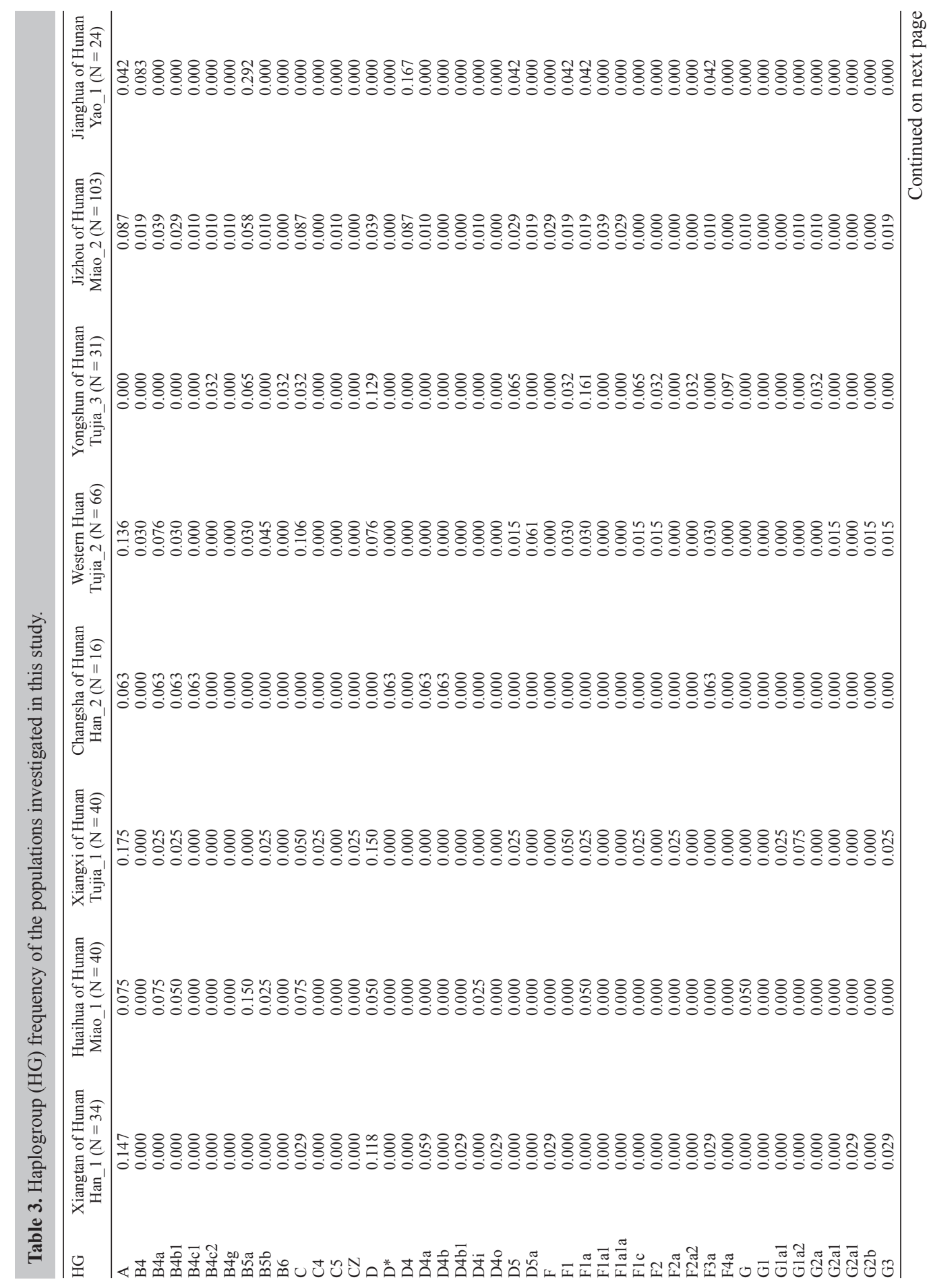


Genetic affinities of the central China ethnic groups

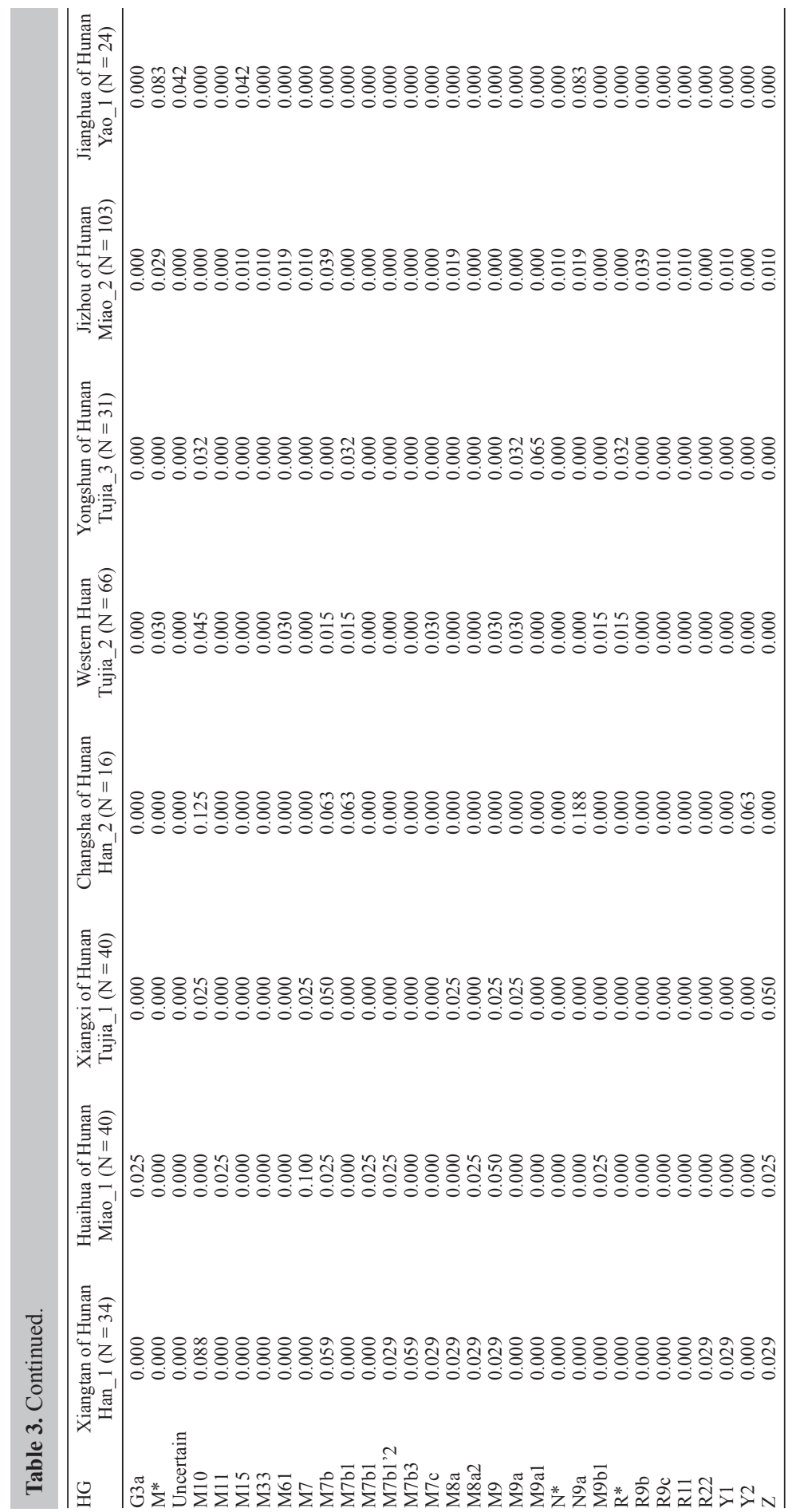


haplogroup frequencies (Table 3) as input factors with the SPSS software (version 16). In addition, the genetic distance and haplotype diversity were evaluated with the Arlequin software package (version 3.1).

\section{RESULTS AND DISCUSSION}

With the updated East Asia and world mtDNA phylogeny tree, all the individuals were assigned to the specific East Asia and southeast Asia haplogroups (Table 2 and Table S1) according to the combined information from control-region and partial coding-region information. The northern East Asia prevalent haplogroup (54.39\%, 62/114, including A, D, C, G, M8, Y, and Z), more than the southern Asia prevalent haplogroup $(41.23 \%, 47 / 114$, containing B, F, M7, M9, and R22), contained the major maternal components of the 3 investigated Hunan populations, which revealed the genetic admixture results of the Hunan populations. However, the 3 populations showed quite different maternal components. In detail, southern China prevalent haplogroups accounted for two thirds $(\sim 60 \%)$ of the maternal components of the Hunan Miao ethnic group, and the Hunan Han and Tujia populations accounted for the remaining third $(\sim 30 \%)$. The northern East Asia prevalent haplogroups were opposite, indicating that the Tujia populations had a higher frequency of northern-eastern Asia prevalent maternal components. The maternal components of the 3 investigated populations was in line with their language branch affinity; the Miao population in Hunan Province belonging to Hmong-Mien language had much higher maternal components popular in southern East Asia than the populations of Han, belonging to Tibeto-Burma language groups. In addition, our results supported that the Tujia of Tibeto-Burma languages group in the Hunan Province may have much closer affinity with the Han populations than the Miao and Yao ethnic groups with the Hmong-Mien language.

To obtain further insight into the affinity between the maternal components and their languages, we compared our datasets with the mtDNAs of the Han, Tujia, Miao, and Yao groups reported in the literature (Wen et al., 2004a,b, 2005). We found that the Yao ethnic groups showed a higher southern of East Asia contribution. This was similar with the Miao population in our dataset; however, the Miao ethnic in literature has a similar contribution from both northern and southern of East Asia maternal contributions. Rather, the 2 Tujia ethnic groups of Tibeto-Burma language branch had a relative higher southern East Asia maternal contribution by comparing with the Tujia population in this study (Figure 2). This implied that the Tujia population had a much more complex prehistory because of its mixture with surrounding populations. Figure 2 showed the principal component map for the first two principal components, which together accounted for $57.71 \%$. In the principal component map, a quite clear clustering pattern between the populations and their language affinity was observed along the diagonal line. In detail, the populations of Hmong-Mien and Han language branch located at two different sides of the fitted line, with $\mathrm{R}$ at 0.063 , and the Tujia populations of Tibeto-Burma family situated at middle between the populations of the Hmong-Mien and Han language families. This clustering pattern was significantly in line with their language affinity, although the populations of the same language family had great maternal genetic differences. The sharing southern of Eastern Asia components of haplogroups B, F, M7, and M9 of the Yao and Miao populations, as well as the A, C, D, G, and Z of the Han and Tujia populations, contributed to the separated clustering affinity. 


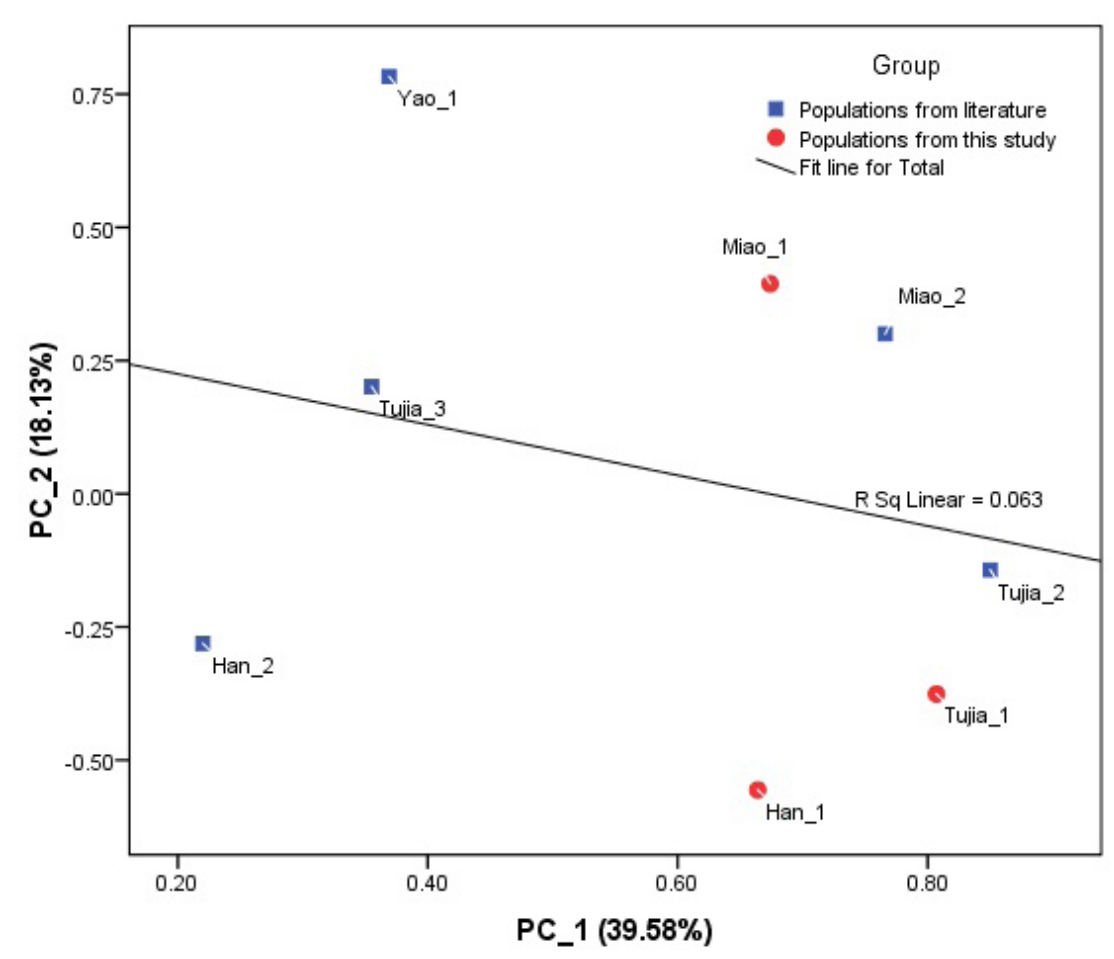

Figure 2. Principal component (PC) analysis of the populations under study.

To better understand the genetic affinities between the populations, the haplotype diversity and the F statistics were performed based on the haplotype frequencies at the significance level of 0.05. Except the lower haplotype diversity in the Yao population, we found similar haplotype diversities among the Han, Tujia, and Miao populations (Table 4). This result supported the plausible genetic admixture results with the immigration from the north of China and the aboriginal southern of China components, which was in line with the history record (Du and Ye, 1994). The relatively lower $F_{\text {ST }}$ value indicated a closer genetic affinity between the Tujia and Han populations than between the Tunjia and Miao populations. However, the Yao ethnic group had the greatest $F_{\mathrm{ST}}$ value with its surrounding populations, matching well with the haplotype estimation results; however, this may be in line with the higher frequency of B and D4 haplogroups in Yao populations, which accounted more than half of the maternal components of the Yao population (Table 5).

Table 4. Haplotype diversity of the populations in this study.
\begin{tabular}{lcccccccc}
\hline Statistics & Han_1 & Han_2 & Miao_1 & Miao_2 & Tujia_1 & Tujia_2 & Tujia_3 & Yao_1 \\
\hline Sample size & 34 & 16 & 40 & 103 & 40 & 66 & 31 & 24 \\
No. of alleles & 33 & 14 & 34 & 82 & 31 & 56 & 28 & 14 \\
Haplotype diversity & $0.9982 \pm$ & $0.9833 \pm$ & $0.9923 \pm$ & $0.9933 \pm$ & $0.9808 \pm$ & $0.9944 \pm$ & $0.9935 \pm$ & $0.9130 \pm$ \\
& 0.0077 & 0.0278 & 0.0073 & 0.0028 & 0.0125 & 0.0039 & 0.0100 & 0.0397 \\
\hline
\end{tabular}

Significance level $=0.0500$. 
Table 5. F-statistic results from haplotype frequencies in the populations of this study.

\begin{tabular}{llllccccc}
\hline & Han_1 & Han_2 & Miao_1 & Miao_2 & Tujia_1 & Tujia_2 & Tujia_3 & Yao_1 \\
\hline Han_1 & 0 & - & - & - & - & - & - \\
Han_2 & 0.0053 & 0 & - & - & - & - & - \\
Miao_1 & 0.00034 & 0.00734 & 0 & - & - & - & - \\
Miao_2 & 0.00257 & 0.00896 & 0.00281 & 0 & - & - & - \\
Tujia_1 & 0.00543 & 0.01493 & 0.00413 & 0.00389 & 0 & - & - \\
Tujia_2 & -0.0003 & 0.00708 & 0.00248 & -0.00005 & 0.0029 & 0 & - \\
Tujia_3 & 0.00032 & 0.00538 & 0.00386 & 0.00093 & 0.00324 & -0.00083 & 0 & - \\
Yao_1 & 0.0365 & 0.04541 & 0.04027 & 0.03299 & 0.05209 & 0.04021 & 0.04481 & 0 \\
\hline
\end{tabular}

Significance level $=0.0500$.

In summary, our extensive analysis on the mtDNAs of the Han, Miao, Yao, and Tujia populations led to further insight into the maternal genetic structure of central China and a better understanding of the relationships between their maternal components and their languages. The formation and development of the central populations was a complex process, affecting each population by integrating genetic components from Han, Tibeto-Burma, and HmongMien language families. Furthermore, the observed that genetic profiles were generally consistent with the language affinity for sharing population-specific maternal components.

\section{ACKNOWLEDGMENTS}

We are grateful to all the voluntary donors of DNA samples used in this study. We thank the anonymous reviewers for their helpful comments on the manuscript. Research supported by the Foundation of China Hunan Provincial Science and Technology Department (\#2011TF1011, \#2012SK3275), the Natural Science Foundation of Yunnan Province (\#2011FB106), and the West Light Foundation of The Chinese Academy of Sciences.

\section{Supplementary material}

\section{REFERENCES}

Andrews RM, Kubacka I, Chinnery PF, Lightowlers RN, et al. (1999). Reanalysis and revision of the Cambridge reference sequence for human mitochondrial DNA. Nat. Genet. 23: 147.

Bandelt HJ, Lahermo P, Richards M and Macaulay V (2001). Detecting errors in mtDNA data by phylogenetic analysis. Int. J. Legal Med. 115: 64-69.

Du RF and Ye FS (1994). Ethnic Groups in China. Science Press, Beijing.

Forster P, Cali F, Rohl A, Metspalu E, et al. (2002). Continental and subcontinental distributions of mtDNA control region types. Int. J. Legal Med. 116: 99-108.

Kivisild T, Tolk HV, Parik J, Wang Y, et al. (2002). The emerging limbs and twigs of the East Asian mtDNA tree. Mol. Biol. Evol. 19: 1737-1751.

Kong QP, Yao YG, Liu M, Shen SP, et al. (2003a). Mitochondrial DNA sequence polymorphisms of five ethnic populations from northern China. Hum. Genet. 113: 391-405.

Kong QP, Yao YG, Sun C, Bandelt HJ, et al. (2003b). Phylogeny of east Asian mitochondrial DNA lineages inferred from complete sequences. Am. J. Hum. Genet. 73: 671-676.

Kong QP, Bandelt HJ, Sun C, Yao YG, et al. (2006). Updating the East Asian mtDNA phylogeny: a prerequisite for the identification of pathogenic mutations. Hum. Mol. Genet. 15: 2076-2086.

Kong QP, Sun C, Wang HW, Zhao M, et al. (2011). Large-scale mtDNA screening reveals a surprising matrilineal complexity in east Asia and its implications to the peopling of the region. Mol. Biol. Evol. 28: 513-522.

Pakendorf B and Stoneking M (2005). Mitochondrial DNA and human evolution. Annu. Rev. Genomics Hum. Genet. 6: 165-183. 
Parson W and Bandelt HJ (2007). Extended guidelines for mtDNA typing of population data in forensic science. Forensic Sci. Int. Genet. 1: 13-19.

van Oven M and Kayser M (2009). Updated comprehensive phylogenetic tree of global human mitochondrial DNA variation. Hum. Mutat. 30: E386-E394.

Wen B, Li H, Lu D, Song X, et al. (2004a). Genetic evidence supports demic diffusion of Han culture. Nature 431: 302-305.

Wen B, Xie X, Gao S, Li H, et al. (2004b). Analyses of genetic structure of Tibeto-Burman populations reveals sex-biased admixture in southern Tibeto-Burmans. Am. J. Hum. Genet. 74: 856-865.

Wen B, Li H, Gao S, Mao X, et al. (2005). Genetic structure of Hmong-Mien speaking populations in East Asia as revealed by mtDNA lineages. Mol. Biol. Evol. 22: 725-734.

Yao YG, Kong QP, Bandelt HJ, Kivisild T, et al. (2002). Phylogeographic differentiation of mitochondrial DNA in Han Chinese. Am. J. Hum. Genet. 70: 635-651.

Yao YG, Kong QP, Wang CY, Zhu CL, et al. (2004). Different matrilineal contributions to genetic structure of ethnic groups in the silk road region in china. Mol. Biol. Evol. 21: 2265-2280.

Zhao M, Kong QP, Wang HW, Peng MS, et al. (2009). Mitochondrial genome evidence reveals successful Late Paleolithic settlement on the Tibetan Plateau. Proc. Natl. Acad. Sci. U. S. A. 106: 21230-21235. 